\title{
Análisis del Ciclo de Vida de los Procesos de Recubrimiento Metálico de Termoplásticos
}

\author{
Daniel Garraín ${ }^{1}$, Rosario Vidal ${ }^{1}$, Pilar Martínez ${ }^{2}$ y Carlos Muñoz ${ }^{1}$ \\ (1) GID, Grupo de Ingeniería del Diseño, Dpto. Ingeniería Mecánica y Construcción, \\ Universitat Jaume I, Av. Sos Baynat, s/n, E-12071 Castellón-España (e-mail: garrain@uji.es) \\ (2) AIMPLAS, Instituto Tecnológico del Plástico, Parque Tecnológico de Valencia, \\ C/ Gustave Eiffel 4, E-46980 Paterna, Valencia-España
}

\begin{abstract}
Resumen
El presente estudio tiene como objetivo evaluar el impacto ambiental de los procesos industriales destinados al recubrimiento metálico de materiales termoplásticos más empleados en la industria. Entre estos se considera el revestimiento con pintura conductora, la deposición química y la deposición a alto vacío. La metodología utilizada para ello ha sido la del Análisis del Ciclo de Vida. Los resultados reflejan que el recubrimiento de pintura conductora tiene el mayor impacto ambiental, debido principalmente a la emisión de compuestos orgánicos volátiles. La deposición metálica a alto vacío presenta el mejor perfil medioambiental, por precisar un consumo mínimo de materias primas y energía durante el proceso. Sin embargo, el elevado coste que supone la adquisición de la maquinaria para realizar el recubrimiento de los termoplásticos mediante esta técnica a alto vacío lleva a que el proceso más comúnmente usado sea el de pintado con pintura conductora.
\end{abstract}

\section{Life Cycle Assessment of Metallic Surface Coating Processes of Thermoplastics}

\begin{abstract}
This study focuses on the evaluation of the environmental impact of the most common industrial processes used in metallic coating processes of thermoplastics. Among these, conductive painting, chemical metal deposition and high-vacuum metal deposition are considered. The methodology used was the Life Cycle Assessment methodology. The results show that conductive coating painting has the greatest environmental impact, especially due to the emission of volatile organic compounds. High-vacuum metal deposition exhibits the best environmental performance, with minimal raw material and energy consumptions throughout the process. However, the high cost associated with the purchase of the required equipment for the high vacuum technique have made conductive coating painting the most commonly used process.
\end{abstract}

Keywords: thermoplastics, metallic coating, life cycle assessment, environmental impact 


\section{INTRODUCCIÓN}

A los materiales termoplásticos utilizados en el ámbito de la electrónica se les suele aplicar un recubrimiento metálico superficial tras su conformado. El propósito de esta técnica, motivos estéticos aparte, es la creación de una superficie conductora que mejora la resistencia a la corrosión y el desgaste, además de proporcionar protección contra las interferencias electromagnéticas (IEM) y las descargas electrostáticas (DES).

El objeto del estudio es la elaboración del ACV de los procesos de recubrimiento metálico de materiales termoplásticos más extendidos en la industria, como son el revestimiento con pintura conductora, la deposición química (o, su nombre en inglés, electroless) y la deposición metálica a alto vacío. No obstante, existen otros procesos como la deposición de vapor metálico (físico o químico) utilizados de forma minoritaria. La metodología del ACV es una herramienta sistemática usada para evaluar las cargas ambientales asociadas al ciclo completo de un producto, proceso o actividad, relacionadas con los efectos ambientales derivados del consumo de materias primas y energías, así como las emisiones y residuos generados.

El revestimiento con pintura consiste en el pintado de los materiales plásticos con pinturas conductoras especiales que contienen metales para la protección frente a los problemas anteriormente mencionados. La Figura 1 detalla las etapas del proceso. La primera etapa consiste en aplicar sobre la pieza plástica una capa de imprimación (normalmente de poliuretano con alto contenido en sólidos) mezclada con catalizador y disolvente, para garantizar la adherencia de la pintura conductora. Este proceso se realiza con una pistola aerográfica en una cabina de pintado. Una vez aplicada esta capa, la pieza se coloca en bandejas y se deja secar al aire. Posteriormente se lija y se pule manualmente para eliminar las imperfecciones (exceso de pintura en los cantos, poros, etc.) en las caras vistas de la pieza. A continuación, el pintado con la pintura conductora se realiza en cabinas provistas de cortina de agua o filtros secos para atrapar el exceso de pintura. El proceso finaliza con el secado de la pieza al aire. Las entradas de material corresponden a las pinturas de imprimación y conductora, mientras que las salidas del sistema la forman los restos de las mismas, los lodos de la limpieza y las emisiones de Compuestos Orgánicos Volátiles (COV).

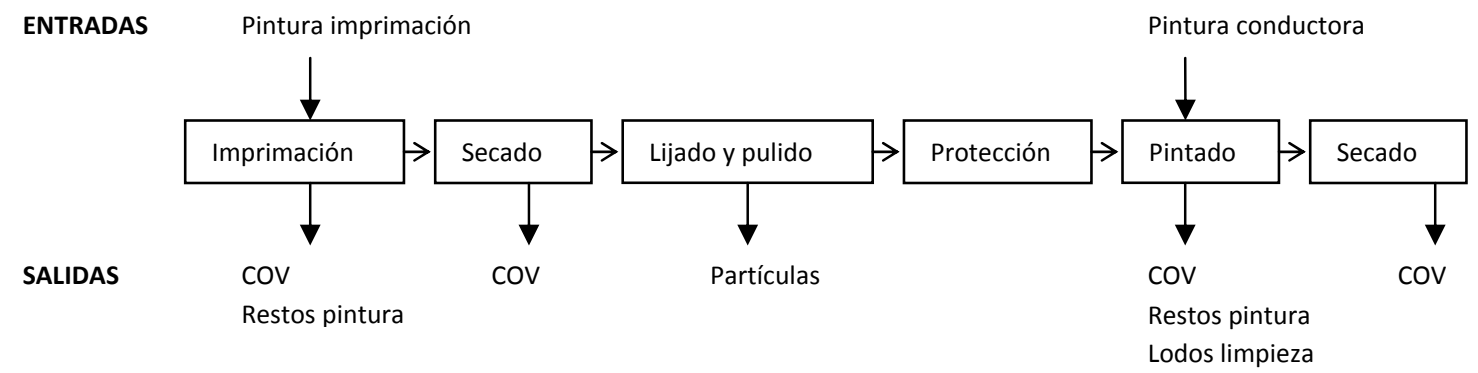

Fig. 1: Diagrama de flujo de materiales en el proceso de pintado conductivo de plásticos

En el proceso de electroless un catión metálico (normalmente de cobre o níquel) en disolución salina se reduce a metal puro (por ejemplo, $\mathrm{Cu}^{+2} \mathrm{a} \mathrm{Cu}^{0}$ ) sobre la superficie del material termoplástico sumergido en la misma. Este proceso no requiere corriente de electrones externa ya que la genera el mismo proceso. En el caso de requerir corriente externa el proceso se denominaría chapado electrolítico (o electroplating). Con la deposición química se consigue recubrir una mayor variedad de piezas que con el chapado electrolítico, pues es mucho más eficiente cuando la pieza plástica posee agujeros o ángulos pronunciados. No obstante, el chapado electrolítico posee mayor eficiencia en piezas con diseño de lengüetas, ranuras o indentaciones en forma redondeada o cónica.

Para llevar a cabo el proceso de deposición metálica a alto vacío, en primer lugar la pieza plástica se limpia a fondo y se le aplica una capa de laca para sellar los poros y rellenar los defectos. A continuación, se graba químicamente con un ácido fuerte para asegurar una buena adherencia del metal, se carga y se deposita en una cámara o crisol donde se produce el vacío. El metal que se deposita sobre la misma (normalmente aluminio, aunque también se realiza con cromo, oro, plata o 
zinc) se alimenta a la cámara y se sublima. Posteriormente, las piezas plásticas comienzan a rotar y es cuando se realiza el vacío, consiguiendo que las moléculas de metal se muevan desde la fuente de evaporación hasta la superficie de la pieza revestir, sin encontrar resistencia del aire y otras partículas gaseosas. Una vez terminado el proceso se libera el vacío, se saca la pieza y se recubre con una laca protectora (Richardson y Lockensgard, 1997). La Figura 2 muestra un esquema del proceso de metalizado.

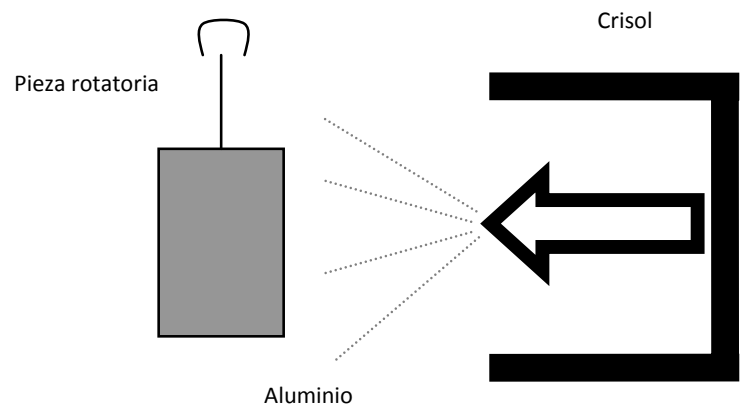

Fig. 2: Deposición metálica a alto vacío de aluminio sobre una película de plástico.

En la literatura hay algunos autores que han realizado estudios del impacto medioambiental de elementos plásticos recubiertos o de distintos procesos de pintado y pinturas. Snowdon (1994), realizó la comparación del impacto medioambiental de distintas placas base de equipamientos de telecomunicaciones formadas por plástico recubierto de metal (mediante electroless), de aluminio y de aluminio parcialmente reciclado, mediante un Análisis del Ciclo de Vida (ACV) en distintos escenarios. Tekawa et al. (1997), han elaborado un estudio destinado a evaluar el impacto ambiental de elementos de los ordenadores personales (PCs) con vistas a desarrollar nuevos componentes de menor carga medioambiental. Los autores consideran el uso de materiales plásticos recubiertos con capa metálica para dicho cometido. Papasavva et al. (2001), han realizado un estudio sobre el impacto medioambiental asociado a diferentes pinturas metalizadas en los procesos de pintado de componentes de vehículos. Dentro del sector de la automoción, Muñoz et al. (2006) han aplicado un ACV a una puerta de un vehículo realizada con material poliolefínico en el que se ha considerado el recubrimiento con pintura conductora. El estudio estaba centrado en el rediseño de dicho componente con materiales reciclados. Finalmente destacar el estudio de Yang et al. (2004), en el que realizan un ACV de teléfonos móviles considerando distintos procesos de recubrimiento como el pintado, la deposición de vapor y el chapado electrolítico. Los autores afirman que este último es el que causa un menor impacto sobre el medio ambiente debido al mayor consumo de energía y de materias primas de la deposición vaporosa.

No obstante, no existen estudios precisos en los que se considere una aproximación del impacto global de los procesos de recubrimiento más utilizados descritos anteriormente, para así poder tener una idea general de cuál de ellos causa un impacto mayor al medio. Por lo tanto, el presente estudio se centra en el ACV de estos procesos. Para ello se han contrastado diversas fuentes de datos de inventario del ciclo de vida considerando los consumos de energía eléctrica y los materiales utilizados. La unidad funcional de medida que se ha tomado en este estudio es $1 \mathrm{~m}^{2}$ de superficie de material plástico revestido.

\section{DATOS DE INVENTARIO DEL CICLO DE VIDA}

En el caso del pintado con pintura conductora, los datos sobre consumos de materia y energía han sido facilitados por una empresa española dedicada al pintado metálico de piezas plásticas y corresponden al tratamiento de una pieza con una superficie de $1275 \mathrm{~cm}^{2}$ y $950 \mathrm{~g}$ de peso. La Tabla 1 muestra los datos inventariados de materia y energía del proceso.

Los datos considerados para realizar el inventario del proceso de deposición química o electroless han sido las cantidades exactas de material utilizado para recubrir el material termoplástico, además del consumo energético. Para calcular la cantidad de materiales se ha considerado un proceso de electroless de níquel, en un baño que contenía cloruro de níquel, hipofosfito sódico e hidróxido 
sódico (Lyman, 1982). Únicamente se ha considerado el desengrasado con carbonato sódico como proceso de pre-tratamiento. La cantidad de materiales necesaria se refleja en la Tabla 2. Para su cálculo se han realizado las siguientes consideraciones: (i) los baños poseen unas dimensiones de 1,5 metros de diámetro x 1,5 metros de altura, para que quepa completamente una pieza de $1 \mathrm{~m}^{2}$, así el volumen de los mismos será de 2650 litros; (ii) el régimen de trabajo será de 2080 horas anuales ( $8 \mathrm{~h} / \mathrm{d}$ áa) y cada pieza está en el baño 3 minutos (para conseguir un espesor de 0,5 micras, ya que la tasa de recubrimiento es de 10 micras/h), por tanto, se tiene una producción de 41600 piezas; y (iii) vaciado completo de los baños cada dos meses. El consumo energético se estima del estudio realizado por la EPA (2002) sobre los procesos de recubrimiento de circuitos impresos por deposición química de cobre, obteniendo $0,43 \mathrm{kWh} / \mathrm{m}^{2}$.

Tabla 1: Entradas y salidas de materia y energía en el pintado de plásticos con pintura conductora

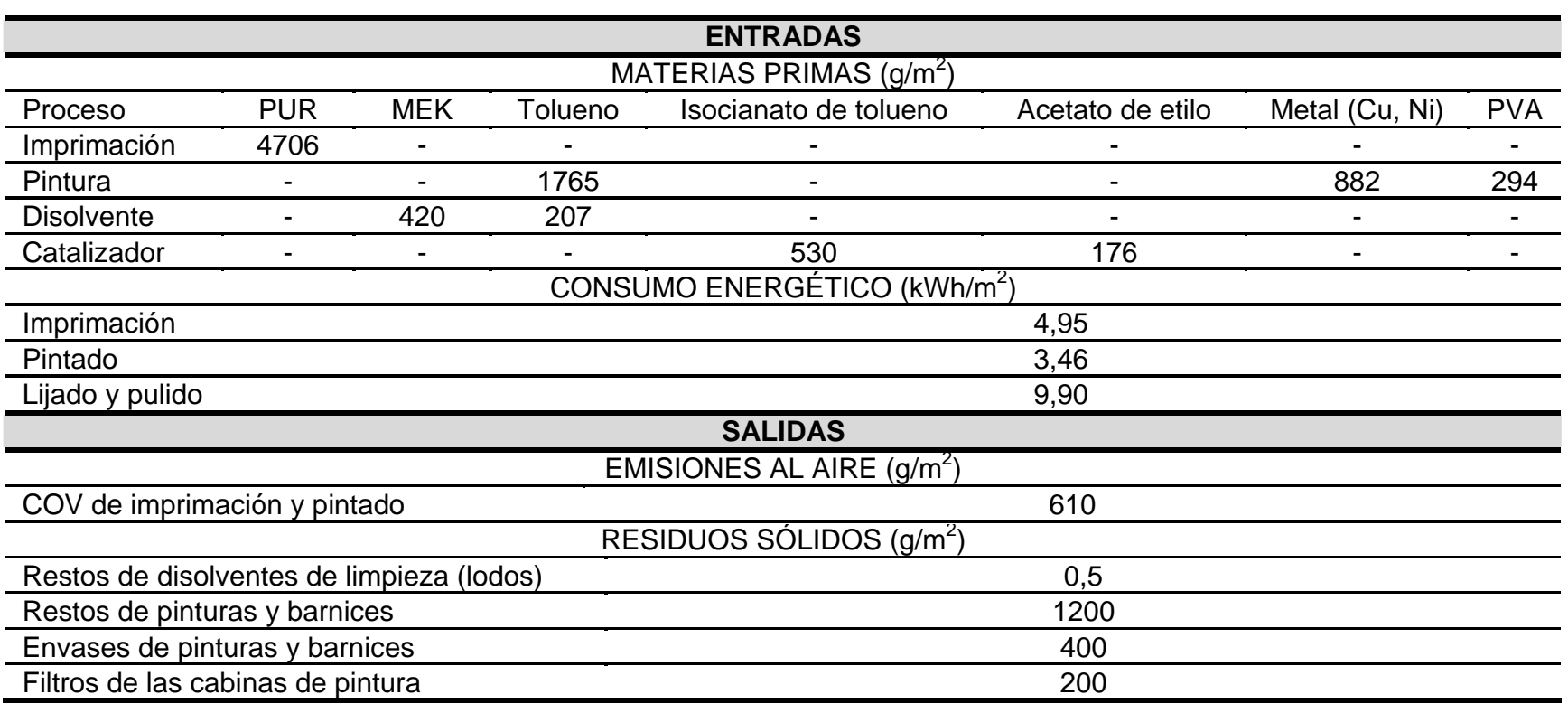

Tabla 2: Consumo de materiales en el proceso de electroless de plásticos

\begin{tabular}{lc}
\hline COMPUESTO & CONSUMO ANUAL $\left(\mathbf{g} / \mathbf{m}^{2}\right)$ \\
\hline Carbonato sódico & 9,56 \\
\hline Cloruro de níquel & 11,47 \\
\hline Hipofosfito sódico & 3,82 \\
\hline Hidróxido sódico & 3,82 \\
\hline
\end{tabular}

Finalmente en el proceso de deposición metálica a alto vacío se ha considerado la cantidad de material de recubrimiento empleado (en este caso, aluminio) y el consumo energético de la máquina. Además se ha tenido en cuenta el consumo de agua de refrigeración. La cantidad de material empleada se ha tomado de un estudio (UPV, 2000) en el que se detalla el proceso de recubrimiento de una película polimérica de $500 \mathrm{~mm}$ de anchura mediante vaporización de aluminio con espesor de capa de 25 micras, obteniendo un consumo de $0,114 \mathrm{~g} / \mathrm{mm}^{2}$ de superficie recubierta. El consumo energético se ha tomado de las especificaciones técnicas de los equipos de metalización por alto vacío de la empresa Kolcer. Considerando un $100 \%$ de eficacia y teniendo en cuenta la superficie a metalizar, la frecuencia del ciclo productivo y la potencia eléctrica absorbida, se calcula el consumo energético medio por superficie de material metalizado, obteniendo un valor de $0,197 \mathrm{kWh} / \mathrm{m}^{2}$. El consumo de agua de refrigeración obtenido es de $9,13 \mathrm{l} / \mathrm{m}^{2}$.

\section{RESULTADOS Y DISCUSIÓN}

Los datos del análisis de inventario de los tres procesos se introdujeron en el programa de evaluación medioambiental comercial SimaPro® ${ }^{\circ} 7.0$ (2004). Se siguió el modelo desarrollado por Guinée (2002) con el conjunto de caracterización y normalización de factores de Europa occidental. En esta fase se llevó a cabo una clasificación de los resultados seleccionando las categorías de impacto más significativas, como son las de eutrofización, acidificación, oxidación troposférica 
fotoquímica, toxicidad humana, calentamiento global y agotamiento de recursos fósiles. Las Tablas 3 y 4 muestran los resultados para cada categoría y la Figura 3 los muestra en forma de porcentaje con respecto al mayor valor.

Tabla 3: Caracterización del impacto medioambiental de los procesos de recubrimiento metálico de termoplásticos por categoría de impacto, por $\mathrm{m}^{2}$ recubierto

\begin{tabular}{lcccc}
\hline CATEGORÍA & UNIDAD & $\begin{array}{c}\text { PINTURA } \\
\text { CONDUCTORA }\end{array}$ & $\begin{array}{c}\text { DEPOSICIÓN } \\
\text { QUÍMICA }\end{array}$ & $\begin{array}{c}\text { DEPOSICIÓN A } \\
\text { ALTO VACÍO }\end{array}$ \\
\hline Eutrofización & $\mathrm{kg} \mathrm{PO}_{4}{ }^{3-} \mathrm{eq}$ & $2,89 \mathrm{E}-02$ & $3,49 \mathrm{E}-05$ \\
\hline Acidificación & $\mathrm{kg} \mathrm{SO}_{2} \mathrm{eq}$ & 0,37 & 0,10 & $7,78 \mathrm{E}-04$ \\
\hline Oxidación fotoquímica & $\mathrm{kg} \mathrm{C}_{2} \mathrm{H}_{4} \mathrm{eq}$ & $1,39 \mathrm{E}-02$ & $1,41 \mathrm{E}-02$ & $2,89 \mathrm{E}-05$ \\
\hline Toxicidad humana & $\mathrm{kg} \mathrm{1,4-DB} \mathrm{eq}$ & 84,48 & 3,54 & $3,42 \mathrm{E}-02$ \\
\hline Calentamiento global & $\mathrm{kg} \mathrm{CO}_{2} \mathrm{eq}$ & 38,36 & 13,14 & 0,11 \\
\hline Agotamiento de recursos & $\mathrm{kg} \mathrm{Sb} \mathrm{eq}$ & 0,44 & 0,12 & $8,45 \mathrm{E}-04$ \\
\hline
\end{tabular}

Tabla 4: Impacto medioambiental normalizado de los procesos de recubrimiento metálico de termoplásticos por categoría de impacto

\begin{tabular}{lccc}
\hline CATEGORÍA & PINTURA CONDUCTORA & DEPOSICIÓN QUÍMICA & DEPOSICIÓN A ALTO VACÍO \\
\hline Eutrofización & $2,32 \mathrm{E}-12$ & $5,20 \mathrm{E}-13$ & $2,80 \mathrm{E}-15$ \\
\hline Acidificación & $1,37 \mathrm{E}-11$ & $3,73 \mathrm{E}-12$ & $2,85 \mathrm{E}-14$ \\
\hline Oxidación fotoquímica & $1,68 \mathrm{E}-12$ & $1,70 \mathrm{E}-12$ & $3,50 \mathrm{E}-15$ \\
\hline Toxicidad humana & $1,12 \mathrm{E}-11$ & $4,68 \mathrm{E}-13$ & $4,52 \mathrm{E}-15$ \\
\hline Calentamiento global & $7,98 \mathrm{E}-12$ & $2,73 \mathrm{E}-12$ & $2,34 \mathrm{E}-14$ \\
\hline Agotamiento de recursos & $2,96 \mathrm{E}-11$ & $8,13 \mathrm{E}-12$ & $5,69 \mathrm{E}-14$ \\
\hline
\end{tabular}

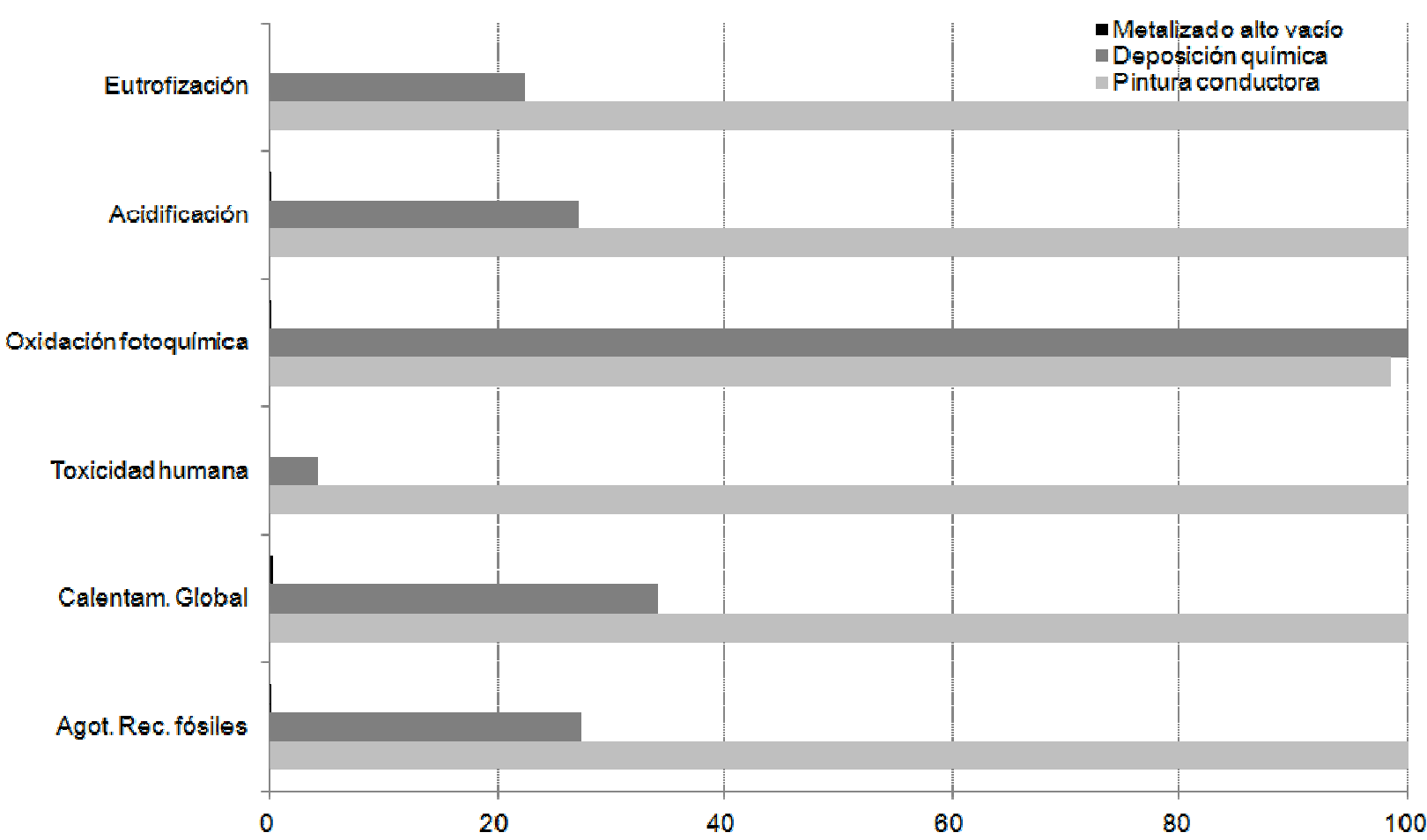

Fig. 3: Eco-perfil comparativo de los procesos de recubrimiento de termoplásticos.

A la vista de los resultados de la tabla 3 y la figura 3, el pintado con pintura conductora es el proceso que mayor impacto sobre el medio genera. Por otra parte, el proceso de deposición metálica a alto vacío presenta un impacto mínimo en comparación con los otros dos procesos. En el primer caso, el mayor consumo energético y las emisiones de COV son los causantes de que la carga medioambiental sea elevada, al contrario que en la deposición al vacío que tiene un mínimo consumo energético y material. Considerando los valores normalizados de las categorías en la tabla 4, la que posee una mayor repercusión es la de agotamiento de recursos fósiles, debido principalmente a los consumos energéticos. 


\section{CONCLUSIONES}

El proceso de recubrimiento de materiales termoplásticos mediante pintado con pintura conductora es el que presenta una carga medioambiental mayor en comparación con otros procesos. El consumo de energía puede considerarse como un "punto caliente" en donde llevar a cabo estrategias de mejora para reducir el consumo de recursos naturales, que llevará consigo además, la reducción del impacto en otras categorías asociadas como el calentamiento global.

El depósito de metal mediante aplicación a alto vacío es el que posee un menor impacto. No obstante, el elevado coste que supone la adquisición de la maquinaria para realizar el recubrimiento de los termoplásticos mediante este proceso lleva a que el proceso más extendido sea el de pintado con pintura conductora.

La metodología del ACV es una herramienta efectiva para determinar la carga ambiental que pueden causar los productos, procesos o servicios. Esta concepción global o filosofía del ciclo de vida (LCT, de sus siglas en inglés Life Cycle Thinking) debería ser difundida en el ámbito empresarial e industrial, tanto a diseñadores y directivos como al personal, para así tener en cuenta, en el momento de la toma de decisiones, todos recursos consumidos y todos los impactos ambientales y sobre la salud que están asociados al ciclo de vida.

\section{REFERENCIAS}

EPA; Alternative technologies for making holes conductive - Cleaner technologies for printed wiring board manufacturers, Report of the U.S. Environmental Protection Agency: EPA-744-R-98-002, USA, Abril (2002). [http://www.epa.gov/dfe/pubs/pwb/pdf/ctsawire.pdf]

Guinée, J. B. (ed); Handbook of Life Cycle Assessment: An operational guide to the ISO standards, 395-658, Kluwer Academics Publishers, Dordrecht, Holanda (2002).

Lyman, T.; Metals Handbook. Surface, Cleaning, Finishing and Coating, Ninth edition, Vol.5, American Society for Metals, Ohio, USA (1982).

Muñoz, I. y otros tres autores; Using LCA to assess Eco-desing in the automotive sector, International Journal of Life Cycle Assessment: 11(5), 323-334 (2006).

Papasavva, S., y otros tres autores; Characterization of automotive paints: an environmental impact analysis, Progress in Organic Coatings: 43, 193-206 (2001).

Richardson, T.L. y E. Lokensgard; Industria del plástico. Plástico industrial, ed. Thomson-Paraninfo, Madrid, España (1997).

SimaPro ${ }^{\circledR}$ software version 7.0, PRé Consultants, Amersfoot, Holanda (2004).

Snowdon, K.G.; Environmental Life Cycle Assessment of Face Plates used in the Telecommunications Industry, Proceedings of the International Symposium of Electronics and the Environment, ISEE, San Francisco, CA, USA (1994). [http://ieeexplore.ieee.org/xpls/ abs_all.jsp?arnumber=337242].

Tekawa, M., S. Miyamoto y A. Inaba; Life Cycle Assessment; An approach to environmentally friendly $P C$, Proceedings of the International Symposium of Electronics and the Environment, ISEE, San Francisco, CA, USA (1997). [http://ieeexplore.ieee.org/xpl/RecentCon.jsp?punumber=4806].

UPV; Acabados superficiales de plásticos y compuestos, Curso de la Universidad Politécnica de Valencia, España (2000).

Yang, J., y otros tres autores; Life cycle assessment of mobile phone housing, Journal of Environmental Sciences: 16(1), 100-103 (2004). 